\title{
Antipsychotic treatment in patients with dementia receiving palliative care: efficiency, fall risk and cognitive impact
}

\author{
Tudor Florea, Elena-Rodica Popescu, \\ Matei Palimariciuc, Roxana Chiriță
}

Tudor Florea - M.D., PhD. student, "Grigore T. Popa" University of Medicine and Pharmacy, Psychiatrist, "Sf. Sava” Hospital, Iași, Romania

Elena-Rodica Popescu - M.D., PhD Student, assistant professor Grigore T. Popa University of Medicine and Pharmacy, Iași; Psychiatrist, Socola Institute of Psychiatry, Iași

Matei Palimariciuc - M.D., PhD Student, assistant professor Grigore T. Popa University of Medicine and Pharmacy, Iași; junior psychiatrist, Socola Institute of Psychiatry, Iași

Roxana Chiriță - M.D., PhD, Professor of Psychiatry Grigore T. Popa University of Medicine and Pharmacy, Iași, Senior psychiatrist Socola Institute of Psychiatry, Iași

\begin{abstract}
Antipsychotics are widely used in treatment of elderly related psychiatric disorders such as delirium, organic hallucinosis. The long-term uses of such drugs are related with increased risks. The objective of this study is to determine efficiency of small doses of antipsychotics in relieving target symptoms and monitoring short-term use in patients diagnosed with dementia receiving palliative care. The study was conducted in a clinical department for palliative care reserved for patients with severe dementia over a period of 1 year. The study sample comprised 56 patients, 31 of which received antipsychotics. Measures used included MiniMental State Exam (MMSE), Neuropsychiatric Inventory Nursing Home Version (NPI-NH), Fall-Risk (FR) Scale, patient records, medication records and fall incidents. We found a significant improvement on NPI-NH Scale $(p<0.05)$ for patients that received antipsychotics and had psychosis, agitation, aggressiveness in accordance with clinical observations. There were no significant changes for $\operatorname{MMSE}(p=0.56)$ or FR Scale $(p=0.87)$. Small doses of antipsychotics have a beneficial impact in treating psychosis and agitation with aggressive behavior in patients with Dementia receiving palliative care. The study showed that the use of antipsychotic medication doesn't correlate with increased cognitive degradation or fall risk. The particularities of these cases need to be further explored.
\end{abstract}




\section{Antipsychotics, dementia, palliative care, risks.}

\section{INTRODUCTION}

Dementia is a progressive debilitating disease that has a devastating impact on the physical, emotional and financial aspect of patients, their families, caregivers and society. The major outcome of this progressive cognitive decline is the inability to function independently. (1) Due to its progressive nature, patients with dementia gradually lose autonomy, severe dementia being characterized by loss of capacity to provide self-care in daily activities, such as maintaining the body hygiene, drinking, eating, disturbance in gait, coordination, urinary and fecal incontinence. $(2,3)$

With the increase of life expectancy, the prevalence of dementia is projected to double every 20 years to approximately 82 billion in 2030 and 152 million by 2050. Worldwide, around 50 million people have dementia, yearly around 10 million new cases are reported. (4)

Severe dementia is often associated with many accumulated co-morbidities and require complex medical care. In contrast to cancer which usually follows an initial slow overall decline from high level of function, dementia follows a prolonged dwindling. (5) The need for the use of end of life care in both diseases have been shown to carry equal importance, thus having a great impact in the shifting strategies for dementia. (6) Many of these patients do not receive palliative care routinely, the main barrier for assessment of symptoms and providing adequate treatment being the inability to communicate. A multidisciplinary approach for neurodegeneration is necessary in developing strategies for future research. As a relatively new field, a solid evidence base for different aspects of dementia in palliative care is required in order to progress the current knowledge. (7)

However, the entire research framework needs to take into account the realities of care towards end-of-life and the frailty of these patients. In the process of studying different aspects of palliative care for dementia, the wellbeing of the patient take priority, thus limiting the usual tools for systematic investigation.

Antipsychotic drugs are widely used for various psychiatric disorders in patients with dementia. Precipitating factors play a key role in the acceleration of neurodegenerative processes; thus, the correct management of such disorders is of prime importance for the evolution of dementia. (8) Delirium is one of the most common causes for admission to a palliative care unit with higher rates seen at the end of life. (9) Also, it is one the most frequent reasons for hospital admittance of patients older than 65 years, with numbers rising over $50 \%$. (10) Until recently, atypical antipsychotics were used in the treatment of delirium, and other psychosis replacing the old "golden standard" Haloperidol. Numerous studies supported the assertion that Risperidone, Olanzapine and Quetiapine had the best results for delirium treatment. (11) Later studies showed a superior response to placebo over antipsychotics, when participants receive individualized nonpharmacologic measures, such as hydration, presence of family, reorientation, vision and hearing aids. $(12,13)$

Even though these facts are still to be proven right, antipsychotics play a key factor in treatment of agitation or psychosis in patients with dementia. Prevalence of psychosis 
among patients with Alzheimer's disease was reported to be $41 \%, 36 \%$ for delusions and $18 \%$ for hallucinations. (14) For patients in nursing home setting hallucinations and delusions are $22 \%$ and $14 \%$ of study subjects respectively. The persistence of symptoms range between 13\%-66\% for delusions and $25 \%-100 \%$ for hallucinations. Among individuals, $36 \%$ have signs of agitation, and $32 \%$ have aggressive behavior, the persistence of these symptoms ranging between 53\%$75 \%$. (15) In choosing the right antipsychotic treatment, expert opinion survey data indicate a higher propensity to Risperidone, Quetiapine and Olanzapine with the indication to avoid other psychiatric drugs in order to avoid more serious side effects. Also, the optimal duration of treatment ranges between 4 to 6 months for dangerous psychosis and dangerous agitation. For lesser agitation and danger, the dosage maintenance indication is in range of 1 to 3 months, afterwards taper and withdrawal. (16)

Conclusive data for patients with dementia in palliative care having antipsychotic treatment is lacking, even though effective strategies for such individuals, suffering of dangerous psychosis and agitation are needed.

\section{METHODS}

\section{Study setting}

The study has been conducted with the approval of Research Ethics Commission of University of Medicine and Pharmacy "Grigore T. Popa" Iasi. Prior to participating in the study, a first-degree relative or legal guardian signed an informed consent.

The present study was a 1-year single center, comparative study to assess the possible outcomes of cognition and fall risk after treatment with antipsychotic medication in therapeutic range for psychosis and severe agitation in patients with dementia in palliative care.

Patients' data was collected at the moment of admission. Mini-Mental State Examination (MMSE), Neuropsychiatric Inventory Nursing Home Version (NPI-NH) and Fall Risk (FR) Scale was performed on every new admission to the study with a reevaluation after 4 weeks. In cases of diagnosed psychosis or extreme agitation, according with International Classification of Diseases, $10^{\text {th }}$ Revision (ICD-10) diagnosis criteria and NPI$\mathrm{NH}$ score, antipsychotic medication was used. Except for antipsychotic medication no other medical intervention or medication change was performed on the selected lot. During the treatment period, patients received either Quetiapine (25-200mg) or Risperidone (0,25$2 \mathrm{mg}$ ). The doses were adapted to patient's weight, in order for the symptoms mentioned to be remitted. The study's design had no intention to compare antipsychotics or to determine dose efficiency.

\section{Participants}

Screening and demographic measurements included diagnosis of dementia according to ICD-10 criteria, anti-dementia medication, sex, age, weight, alcohol and tobacco consumption, medical and surgical history, full physical examination, laboratory analysis, and electrocardiogram. Inclusion criteria were: diagnosis of dementia with a MMSE score under 14, need for palliative care, treatment with anti-dementia medication and age over 65 years old. Exclusion consisted of prior history of psychiatric diagnosis.

Fifty-six patients that met the criteria were hospitalized during this period. From this lot, thirty-one patients received antipsychotics.

\section{Test batteries and statistics}

For the assessment of psychosis, agitation and aggressive behavior Neuropsychiatric 
Inventory Nursing Home Version (NPI-NH) scale was used at two distinctive moments, at the moment of entrance to the study and after 4 weeks. This instrument assesses the neuropsychiatric symptoms and psychopathology of patients with dementia, the information being gathered by professional caregivers. The test includes ten behavioral subsections and two types of neurovegetative changes. The subsections have each one frequency and one severity aspect built into the questionnaire. (17)

For the assessment of dementia and for screening purposes Mini-Mental State Examination (MMSE) was used at two distinct moments, at the moment of study entering and after 4 weeks. MMSE is a cognitive test that is commonly used in the diagnostic and assessment of dementia. (18) In order to assess the fall risk, we used a scale developed at "Socola" Institute of Psychiatry with ten items that account for fall events in latest month, disorientation, anxiety and agitation, loss of hearing and sight, balance and gait, use of opioids and sedative medication, age older than 65 years old, other symptoms connected with legs (pain, numbness, history of injury, surgical procedures, or other disorders) and the need for medical gait-assisting devices. Each of the items have a $1 / 0$ value, with the final result evaluating the fall risk. The scale development had as a basis the Downton fall risk index. (19)

Statistics was performed using analysis of variance for repeated measures (ANOVA). Statistic differences were tested first on the entire sample for each test, regardless of use of antipsychotics. Afterwards, the sample was split in treated "with-antipsychotics" and "without-antipsychotics" and afterwards tested for statistical significance.

\section{RESULTS}

Results and statistics are summarized in Table 1. Fifty-seven participants met the inclusion criteria, 31 received antipsychotics (9 receiving Quetiapine, 19 receiving Risperidone, 2 receiving Tiapride), 25 not meeting criteria for treatment with antipsychotics (had a low score on NCI-NH and didn't met the criteria for diagnostic). The patients were spread on a normal distribution chart with an average age of 82 years with a standard deviation (S.D) of 7.5 years. Patients with treatment had an average of 80.5 years and without treatment an average of 84.3 years, with S.D. of 7.5 and 7.0 respectively. (Fig. 1)

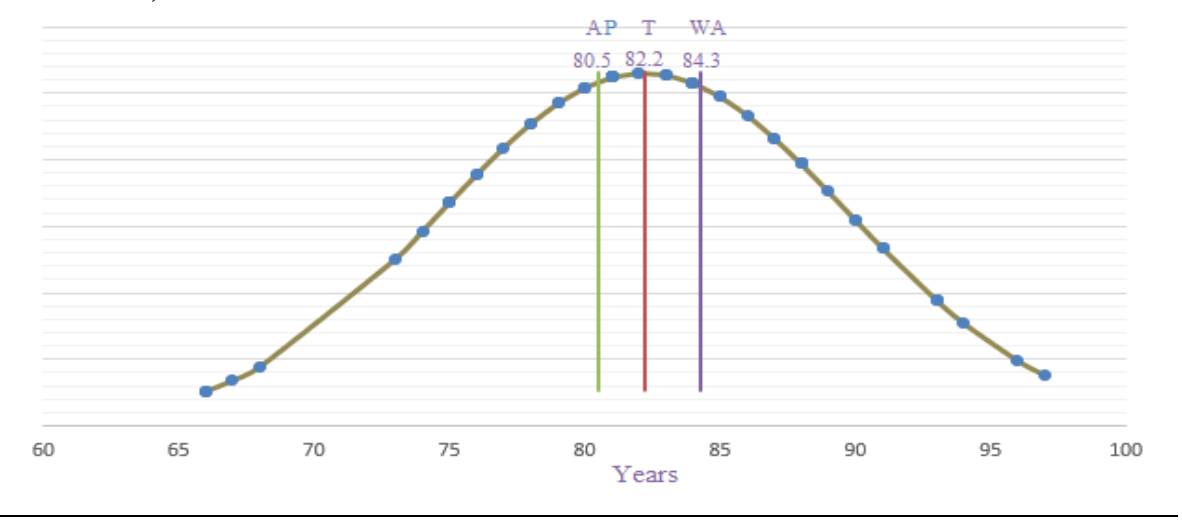

Figure 1. Normal distribution of age average

AP: Patients with antipsychotics, T: Total number of patients

WA: Patients without antipsychotics 
Bulletin of Integrative Psychiatry O New Series $\bigcirc$ December 2019 ○ Year XXV ONo. 4 (83)/83 s The oldest patient in our study had 97 years old, and the youngest 66. The oldest patient that received antipsychotics had 86 years old and the youngest 66 . None of the groups had any extrapyramidal symptoms (EPS), a and a number of three patients manifested light vertigo (two in antipsychotics group) for one to two days. Two patients had a diagnostic of Parkinson disease, one was number of 2 patients of the "withoutantipsychotics" group had a urinary infection

\begin{tabular}{|l|l|l|l|l|l|l|l|l|}
\hline \multicolumn{7}{|c|}{ Table I. Demographic data - Age (years) } \\
\hline & N & Min & Q1 & Q2 & Q3 & Max & Average & S.D. \\
\hline Total & 56 & 66 & 78 & 82 & 88 & 97 & 82.19 & 7.51 \\
\hline Females & 34 & & & & & & 82.88 & 7.3 \\
\hline Females-AP & 17 & & & & & & 80.2 & 7.22 \\
\hline Males & 22 & & & & & & 81.13 & 7.7 \\
\hline Males-AP & 14 & & & & & & 80.8 & 6.8 \\
\hline AP: Patients with antipsychotics, S. D.: standard deviation \\
\hline \multicolumn{78}{|l|}{} \\
\hline
\end{tabular}

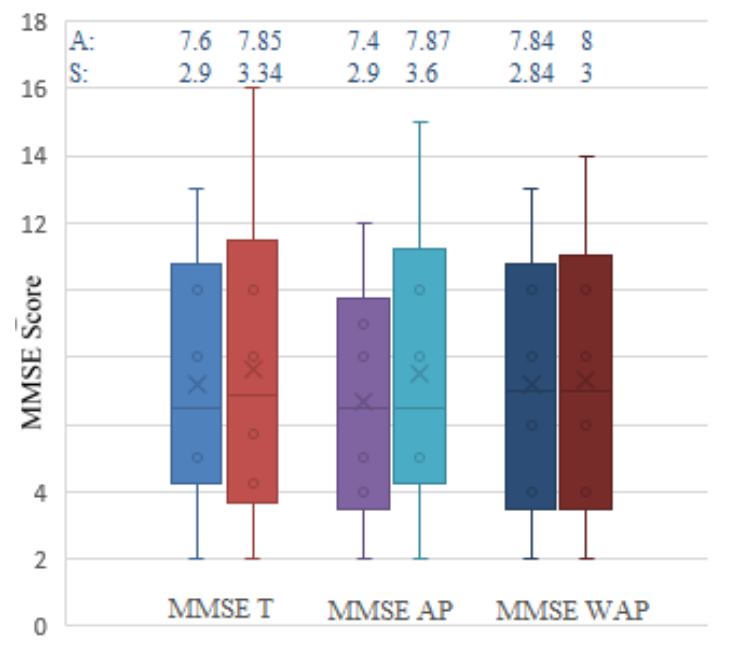

Figure 2. Interquartile Range - Mini Mental State Examination

MMSE T: total number of patients, AP: patients with antipsychotics, WAP: patients without antipsychotics, A: Average score, S: Standard Deviation

Between groups, the average testing score varied between 7.4 and 7.84 for MMSE and between 5.9 and 6.28 for Fall Risk. An overall slight improvement of the MMSE Score was noticed for patients that received antipsychotics. (Fig. 2) It was noticed a slight reduction of the Fall Risk Score averages between the two groups. (Fig. 3) This small improvement was further tested in order to find a statistical significance; the results disproved our hypothesis ( $p>0.05)$. (Table II)

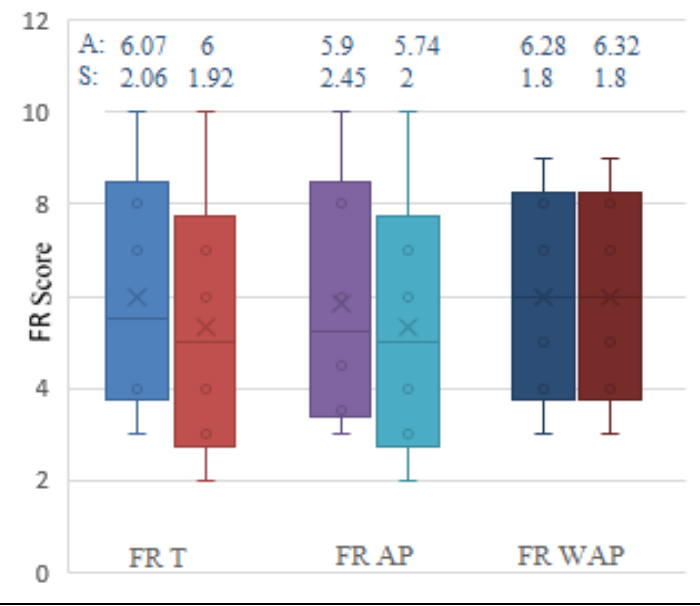

Figure 3. Interquartile Range - Fall Risk score

FR T: total number of patients, AP: patients with antipsychotics, WAP: patients without antipsychotics, A:

Average score, S: Standard Deviation

For the NPI-NH Score, the first data showed a noticeable improvement in score averages in total participants (Average 1st test 24.3 Average 2 nd test 10.6). When focused on the two groups (with/without antipsychotics AP/WAP) the averages dropped visibly for 
the "with-antipsychotics" group (Average from 35.1 to 12 ), and only a small difference for the group "without-antipsychotics" (Average from 11.4 to 8.9). In order to support these findings further statistical testing was performed.

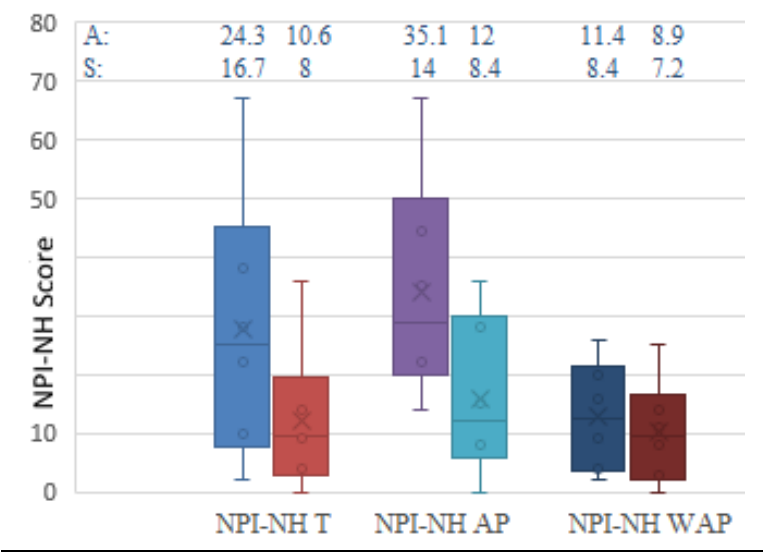

Figure 4. Interquartile Range for Neuropsychiatric Inventory Nursing Home Version (NPI-NH)

NPI-NH T: total number of patients, AP: patients with antipsychotics, WAP: patients without antipsychotics A: Average score, S: Standard Deviation
Antipsychotic medication reduced the NPINH Score $(-0.05 ; 95 \% \quad C I, p=2.48 E-07)$ assessed for the entire group, and for the "with-antipsychotic" group $(-0.05 ; 95 \% \mathrm{CI}$, $\mathrm{p}=5.9 \mathrm{E}-11)$. When tested, the "withoutantipsychotic" group the results were not statistically significant $(-0.05 ; 95 \% \quad \mathrm{CI}$, $\mathrm{p}=0.27$ ).

\begin{tabular}{|c|c|c|c|c|c|}
\hline \multicolumn{6}{|c|}{ Table II. The primary data for MMSE, FR and NPI-NH } \\
\hline ANOVA & & & & & \\
\hline Source of Variation: $M M S E$ & $\mathbf{N}$ & $M S$ & $\boldsymbol{F}$ & P-value & F crit \\
\hline Between Groups Total & 56 & 2.58 & 0.257 & 0.613 & 3.92 \\
\hline \multicolumn{6}{|l|}{ Source of Variation: FR } \\
\hline Between Groups Total & 56 & 0.14 & 0.035 & 0.85 & 3.93 \\
\hline Between Groups WAP & 25 & 0.02 & 0.006 & 0.94 & 4.04 \\
\hline Between Groups AP & 31 & 0.12 & 0.03 & 0.87 & 3.92 \\
\hline \multicolumn{6}{|l|}{ Source of Variation: NPI-NH } \\
\hline Between Groups Total & 56 & 5293.75 & 30.26 & $2.5 \mathrm{E}-07$ & 3.927 \\
\hline Between Groups WAP & 25 & 79.38 & 1.24 & 0.27 & 4.04 \\
\hline Between Groups AP & 31 & 8314.9 & 63.24 & $5.9 \mathrm{E}-11$ & 4.0 \\
\hline \multicolumn{6}{|c|}{$\begin{array}{l}\text { MMSE: Mini-Mental State Examination, FR: Fall Risk Score, NPI-NH: Neuropsychiatric Inventory } \\
\text { Nursing Home Version, S.D.: Standard Deviation, Total: Total number of patients, WAP: Patients } \\
\text { that had no antipsychotics, AP: Patients that received antipsychotics }\end{array}$} \\
\hline
\end{tabular}


Antipsychotics didn't improve MMSE Score $(-0.05 ; 95 \%$ CI, $\mathrm{p}=0.61$; F $<$ Fcrit $)$ or FR Score $(-0.05 ; \quad 95 \% \quad$ CI, $\quad \mathrm{p}=0.85 ; \quad \mathrm{F}<$ Fcrit $)$ respectively. (Table II)

For statistical significance F-Test had positive results only for patients "with-antipsychotics" on NPI-NH test and for total sample on NPINH test. The sample "without-antipsychotics" had negative F-Test results ( $\mathrm{F}<\mathrm{Fcrit})$.

\section{DISCUSSION}

The use of antipsychotics in elderly people is a common thing. For institutionalized patients, many of whom have dementia, the antipsychotic treatment has an extended use. (20)

During study development, a first issue encountered was the deficiency in objective signs and symptoms evaluation. A correct diagnostic assessment is becoming harder with age and severity of dementia. The items used for testing have a level of subjectivity built in (NPI-NH), thus an extra level of precaution in data gathering was needed.

The present study revealed that the short-term use of low doses of second-generation antipsychotics (Risperidone, Quetiapine) in patients with dementia in palliative care had a positive impact on NPI-NH scale and timid positive influence on MMSE scale (not statistically significant for MMSE). The current results are comparable with other studies for Risperidone and Quetiapine that may be more effective for particular symptoms, such as anger, aggression, and paranoid ideas. They do not appear to improve functioning, or quality of life. (21, 22, 23)

Our testing for cognitive impairment associated with psychosis or aggressive behavior showed no statistical significance before and after the use of antipsychotics followed by the reduction of symptoms as seen in other psychotic disorders. (24) Even though cognition testing may be affected through agitation and deficiencies in communication, we found that the sole use of MMSE doesn't account for a full cognitive test battery in severe dementia.

Dementia is a disease that is very often associated with increased fall risk. Factors that determine lack of equilibrium and gait disorders are numerous, intrinsic and extrinsic, some shared with older adults in general and others unique to the disease. (25) Complications from such events have a powerful impact on overall quality of life, care needs and mortality. (26)

During the study, some patients suffered falling events or loss of equilibrium. None suffered complications, none required interventions. We found no statistical difference between the two groups and no linking between the use of antipsychotics in small dosages and an increased fall risk. In palliative care, the independence of patients is of primordial importance. Lack of movement impairments and self-conducting behavior has a positive impact on overall quality of life, thus assessment for the right use of medication in preventing such deteriorating factors has a great importance in dementia. Costs associated with falling in elderly is another important aspect, thus making informed and documented decisions in the use of antipsychotics a key quality for every practitioner. (27)

The occurrence of other symptoms related to antipsychotics, such as EPS, were not encountered among our subjects. Our study design focused on the development of side effects in patients treated with antipsychotics, 


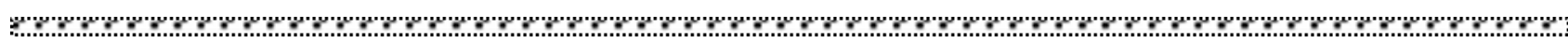
in quantities that we deemed safe for palliative care.

agitation without increasing the risk of falling or other major events, when the dosages of

Our findings showed the possibility of antipsychotics are kept in therapeutic range treating psychosis, aggressive behavior, with constant monitoring.

\section{CONCLUSIONS}

Associated pathology of dementia remains a challenging issue in palliative care for all practitioners. Comorbidities and increased risk factors make the treatment with antipsychotics a continuous struggle to finding the right balance between harm and remedy. Further studies are needed to understand and apply an objective and efficient way for diagnostic and assessment of different symptoms related to old age and severe dementia.

Second generation antipsychotics are useful in treating psychosis, aggressive behavior and agitation in patients with dementia in palliative care. Our study showed no increased fall risk.

Developing more efficient guidelines for the use of antipsychotics in palliative care, thus orienting practitioners in how to tailor and implement effective strategies is needed. Although our data are encouraging, more studies are needed in order to validate our assumptions and account for the varied pathophysiology, combination with other therapies, drugs and different social background.

\section{ACKNOWLEDGEMENTS AND DISCLOSURES}

The authors declare that they have no potential conflicts of interest to disclose.

\section{REFERENCES}

1. Chertkow H, Feldman HH, Jacova C, Massoud F. Definitions of dementia and predementia states in Alzheimer's disease and vascular cognitive impairment: consensus from the Canadian conference on diagnosis of dementia. Alzheimers Res Ther. 2013;5(Suppl 1):S2

2. Wu JM, Matthews CA, Vaughan CP, Markland AD. Urinary, fecal, and dual incontinence in older U S. Adults. J Am Geriatr Soc. 2015; 63 (5):947-953

3. Schafirovits-Morillo L, Suemoto CK. Severe dementia: A review about diagnoses, therapeutic management and ethical issues. Dement Neuropsychol. 2010;4(3):158-164

4. World Health Organisation: Dementia fact sheet. 2017. http://www.who.int/mediacentre/factsheets/fs362/en/.

5. Murray SA, Kendall M, Boyd K, Sheikh A. Illness trajectories and palliative care. BMJ. 2005;330(7498):10071011.

6. Hughes JC, Robinson L, Volicer L. Specialist palliative care in dementia. BMJ. 2005;330(7482):57-58.

7. Fox S, FitzGerald C, Harrison Dening K, et al. Better palliative care for people with a dementia: summary of interdisciplinary workshop highlighting current gaps and recommendations for future research. BMC Palliat Care. 2017;17(1):9.

8. Fong TG, Davis D, Growdon ME, Albuquerque A, Inouye SK. The interface between delirium and dementia in elderly adults. Lancet Neurol. 2015;14(8):823-832.

9. Hosie A, Davidson PM, Agar M, Sanderson CR, Phillips J. Delirium prevalence, incidence, and implications for screening in specialist palliative care inpatient settings: a systematic review. England; 2013 Jun.

10. Inouye SK, Westendorp RG, Saczynski JS. Delirium in elderly people. Lancet. 2014;383(9920):911-922.

11. Markowitz JD, Narasimhan M. Delirium and antipsychotics: a systematic review of epidemiology and somatic treatment options. Psychiatry (Edgmont). 2008;5(10):29-36. 
12. Agar MR, Lawlor PG, Quinn S, et al. Efficacy of Oral Risperidone, Haloperidol, or Placebo for Symptoms of Delirium Among Patients in Palliative Care: A Randomized Clinical Trial. JAMA Intern Med. 2017;177(1):34-42.

13. Boettger S, Jenewein J. Placebo might be superior to antipsychotics in management of delirium in the palliative care setting. Evid Based Med. 2017;22(4):152-153.

14. Ropacki SA, Jeste DV. Epidemiology of and risk factors for psychosis of Alzheimer's disease: a review of 55 studies published from 1990 to 2003. Am J Psychiatry. 2005;162(11):2022-2030.

15. Selbæk G1, Engedal K, Bergh S. The prevalence and course of neuropsychiatric symptoms in nursing home patients with dementia: a systematic review. J Am Med Dir Assoc. 2013 Mar;14(3):161-9.

16. Reus VI, Fochtmann LJ, Eyler AE, et al. The American Psychiatric Association Practice Guideline on the Use of Antipsychotics to Treat Agitation or Psychosis in Patients With Dementia. Am J Psychiatry. 2016;173(5):543-546.

17. Zuidema SU, Buursema AL, Gerritsen, Maarten G J M, et al. Assessing neuropsychiatric symptoms in nursing home patients with dementia: reliability and Reliable Change Index of the Neuropsychiatric Inventory and the CohenMansfield Agitation Inventory. Int J Geriatr Psychiatry. 2011;26(2):127-134.

18. Creavin ST, Wisniewski S, Noel-Storr AH, et al. Mini-Mental State Examination (MMSE) for the detection of dementia in clinically unevaluated people aged 65 and over in community and primary care populations. England; 2016 Jan 13.

19. da Costa BR, Rutjes AWS, Mendy A, Freund-Heritage R, Vieira ER. Can falls risk prediction tools correctly identify fall-prone elderly rehabilitation inpatients? A systematic review and meta-analysis. PLoS One. 2012;7(7):e41061.

20. Kamble P, Chen H, Sherer JT, Aparasu RR. Use of antipsychotics among elderly nursing home residents with dementia in the US: an analysis of National Survey Data. Drugs Aging. 2009;26(6):483-492.

21. Sultzer DL, Davis SM, Tariot PN, et al. Clinical symptom responses to atypical antipsychotic medications in Alzheimer's disease: phase 1 outcomes from the CATIE-AD effectiveness trial. Am J Psychiatry. 2008;165(7):844-854.

22. Schneider LS, Dagerman K, Insel PS. Efficacy and adverse effects of atypical antipsychotics for dementia: metaanalysis of randomized, placebo-controlled trials. Am J Geriatr Psychiatry. 2006;14(3):191-210.

23. Savaskan E, Schnitzler C, Schroder C, Cajochen C, Muller-Spahn F, Wirz-Justice A. Treatment of behavioural, cognitive and circadian rest-activity cycle disturbances in Alzheimer's disease: haloperidol vs. quetiapine. Int $\mathbf{J}$ Neuropsychopharmacol. 2006;9(5):507-516.

24. Sheffield JM, Karcher NR, Barch DM. Cognitive Deficits in Psychotic Disorders: A Lifespan Perspective. Neuropsychol Rev. 2018;28(4):509-533.

25. Fernando E, Fraser M, Hendriksen J, et al. Risk Factors Associated with Falls in Older Adults with Dementia: A Systematic Review. Physiother Can. 2017;69(2):161-170.

26. Burns E, Kakara R. Deaths from Falls Among Persons Aged /=65 Years - United States, 2007-2016. MMWR Morb Mortal Wkly Rep. 2018;67(18):509-514.

27. Stevens JA, Corso PS, Finkelstein EA, Miller TR. The costs of fatal and non-fatal falls among older adults. Inj Prev. 2006;12(5):290-295.

\section{Correspondence:}

Matei Palimariciuc,

MD, PhD student, "Socola” Institute of Psychiatry, Bucium street no. 36, Iași, Romania, 700282, matei.palimariciuc2000@gmail.com

Submission: 01 sep 2019

Acceptance: 24 nov 2019 